\title{
Avaliação da sensibilidade a antimicrobianos de 87 amostras clínicas de enterococos resistentes à vancomicina
}

\author{
I.H. Saraiva, *R.N. J Ones, *M. ERWin, H.S. SAder
}

Laboratório Especial de Microbiologia Clínica, Disciplina de Doenças I nfecciosas e Parasitárias, U niversidade F ederal de São Paulo E scola Paulista de Medicina, São Paulo, SP. *Anti-I nfective Research Center, Departamento de Patol ogia, U niversidade de I owa, I owa City, IA, EUA.

RESUMO - Oвjetivos. 1) Avaliar o padrão de sensibilidade in vitro de amostras clínicas de enterococos resistentes à vancomicina (ERV), aos antimicrobianos comumente utilizados no seu tratamento, bem como a anti microbianos alternativos. 2) Avaliar a acurácia do E test, em comparação aos outros testes de sensibilidade a antimicrobianos (microdiluição em caldo e difusão em disco).

Material e Métodos. Foram analisadas 87 amostras clínicas de ERVs selecionadas de 1.936 isolados de enterococos coletados em 97 hospitais norte-americanos, no último trimestre de 1992. A identificação em nível de espécie foi feita pelos sistemas API 20S, Vitek e uma versão modificada do método convencional proposto por Facklam e Collins. A avaliação da sensibilidade in vitro aos antimicrobianos foi realizada pela técnica de microdiluição em caldo, E test e métodos de difusão em disco. As amostras foram testadas, tanto para antimicrobianos normalmente utilizados no tratamento de infecções enterocócicas (vancomi cina, teicoplanina, ampicilina, penicilina, gentamicina e estreptomicina), como também para drogas alternativas potencialmente úteis (cloranfenicol, doxiciclina, esparfloxacina, ciprofloxacina, clinafloxacina, eritromicina, espectinomi-

\section{NTR ODUÇÃO}

Embora, normalmente, colonizantes do trato gastrointestinal e genital feminino, os enterococos têm-se destacado, nos últimos anos, como patógenos importantes em infecção hospitalar. Dados norte-americanos já os apontam como segunda causa mais comum de infecção nosocomial nos Estados Unidos, estando particularmente associados a infecções do trato urinário, infecções de ferida cirúrgica e bacteremias ${ }^{1}$. Paral elamente ao aumento da incidência, tem-se observado rápido aumento na freqüência de cepas resistentes aos antimicrobianos de uso corrente, mesmo aos glicopeptídeos².

Análise de dados enviados ao Center for Disease Control (CDC), no período de janeiro de 1989 a março de 1993, demonstra um aumento de vinte cina, trospectomicina, trimetoprim-sulfametoxazol e novobiocina).

Resultados. A avaliação dos testes de sensibilidade das 87 amostras de ERV revelou resistência, a ampicilina em torno de $86 \%$, o mesmo sendo observado para penicilina. E m relação aos aminoglicosídeos, obtivemos alto grau de resistência, em torno de $82 \%$ e $85 \%$, para gentamicina e estreptomicina, respectivamente. Apesar de pertencer à mesma classe da vancomicina, a teicoplanina foi ativa contra $29 \%$ das amostras de ERV. Entre os antimicrobianos alternativos testados, os que apresentaram maiores taxas de sensibilidade foram o cloranfenicol, a doxiciclina e a trospectomicina $(82 \%, 92 \%$ e $94 \%$ de isolados suscetíveis, respectivamente).

Conclusão. $O$ tratamento de infecções causadas por enterococos multirresistentes ainda é um desafio, e vários esquemas já vêm sendo propostos na literatura. São necessários, no entanto, mais trabalhos analisando a efetividade clínica dessas combinações de antibióticos antes que recomendações definitivas possam ser feitas.

UNITERMOS: Enterococos resistentes à vancomicina. Atividade antimicrobiana in vitro. Resistência a antimicrobianos. Novos antibióticos. E test.

vezes na prevalência de resistência à vancomicina em enterococos associados com infecções nosocomiais, nos Estados Unidos ${ }^{3}$. Apesar da publicação de vários trabalhos sobre enterococos multirresistentes, pouco ainda se sabe sobre sua forma de disseminação, suas características epidemiológicas e, sobretudo, ainda não se conhece a melhor abordagem terapêutica diante de infecções graves causadas pelos ERVs, os quais, muitas vezes, também se mostram resistentes aos demais antimicrobianos comumente utilizados no seu tratamento4.

No presente estudo, foram avaliadas 87 amostras clínicas provenientes de 97 hospitais norteamericanos. Essas amostras tinham como característica em comum o fato de serem resistentes à vancomicina. Esses isolados foram testados para antimicrobianos normalmente utilizados para o 
tratamento de infecções enterocócicas e, também, para dez drogas alternativas potencialmente úteis, com o intuito de avaliar o padrão de sensibilidade dos ERV e discutir possíveis opções terapêuticas.

\section{MATERIAL E MÉTODOS}

Os 87 isolados resistentes à vancomicina foram selecionados de 1.936 amostras clínicas coletadas e processadas nos três últimos meses de 1992 em 97 hospitais norte-americanos ${ }^{3}$. Essas amostras foram isoladas em sangue ou outros líquidos corpóreos e secreções, exceto urina. Esses 87 i solados correspondem a $4,5 \%$ de todas as amostras aval iadas nesse estudo prévi $0^{3}$ e estavam distribuídos entre 22 hospitais. A identificação em nível de espécie foi feita pelos sistemas API 20S, Vitek e uma versão modificada ${ }^{5}$ do método convencional proposto por Facklam e Collins ${ }^{6}$.

OS ERV foram testados contra vancomicina e tei coplanina por meio de mi crodi lui ção em cal do, $\mathrm{E}$ test e métodos de difusão em disco, e contra ampicilina, penicilina e gentamicina mediante $\mathrm{E}$ test e difusão em disco. Estreptomicina, por sua vez, foi avaliada unicamente por E test. Os testes de sensibilidade foram realizados segundo os critérios do National Committee for Clinical Laboratory Standards (NCCLS) $)^{7,8}$.

Todos os 87 isol ados foram também testados contra dez drogas al ternativas potencialmente úteis no tratamento de infecções enterocócicas. As drogas avaliadas foram: ciprofloxacina, clinafloxacina ${ }^{9}$, esparfloxacina, doxiciclina, eritromicina, espectinomicina, trospectomicina ${ }^{10}$, cloranfenicol, trimetoprim-sulfametoxazol e novobiocina. Essas drogas foram avaliadas pelas técnicas de $E$ test e/ou difusão em disco.

$\mathrm{F}$ oi definido como resistente à vancomicina ou à tei coplanina o isol ado que apresentasse concentração inibitória mínima (MIC) maior ou igual a 32 $\mu \mathrm{g} /$ $\mathrm{mL}$ para esses antimicrobianos. Foram considerados sensíveis à vancomicina os isolados que apresentassem MIC $\leq 8 \mu \mathrm{g} / \mathrm{mL}$ a esse glicopeptídeo, enquanto que a sensibilidade à teicoplanina é definida como MIC $\leq 4 \mu \mathrm{g} / \mathrm{mL}^{7}$. A diferença nos breakpoints de sensibilidades destes dois gl icopeptídeos se deve a diferenças na farmacologia e farmacocinética. Os isolados que apresentaram MIcs entre 8 e $32 \mu \mathrm{g} / \mathrm{mL}$ para vancomicina e entre 4 e $32 \mu \mathrm{g} / \mathrm{mL}$ para teicoplanina foram classificados como intermediários para os respectivos antimicrobianos. Com rel ação à ampicilina, o NCCLS considera resistente o isolado que apresenta MIC $\geq 16 \mu \mathrm{g} / \mathrm{mL}$, e sensível aquele que apresenta $\mathrm{MIC}<16 \mu \mathrm{g} / \mathrm{mL}$, não existindo, assim, a categoria intermediária para essa combinação espécie/antimicrobiano ${ }^{7}$.

\begin{tabular}{|cccc|}
\hline \multicolumn{3}{|l}{$\begin{array}{l}\text { Tabela 1 - Padrão de sensibilidade das } 87 \text { amostras de ERV } \\
\text { de acordo com o método utilizado }\end{array}$} \\
\hline Antimicrobiano* & $\begin{array}{c}\text { Sensível } \\
\mathbf{n}(\%)\end{array}$ & $\begin{array}{c}\text { Intermediário } \\
\mathbf{n}(\%)\end{array}$ & $\begin{array}{c}\text { Resistente } \\
\mathbf{n}(\%)\end{array}$ \\
Teicoplanina & & - & $62(71)$ \\
MIC** & $25(29)$ & $3(3)$ & $59(68)$ \\
E test & $25(29)$ & $5(6)$ & $57(65)$ \\
Disco & $25(29)$ & - & $75(86)$ \\
Ampicilina & $12(14)$ & - & $76(87)$ \\
E test & $11(13)$ & $1(1)$ & $75(86)$ \\
Disco & $11(13)$ & - & $79(91)$ \\
Penicilina & $8(9)$ & - & $71(82)$ \\
E test & & - & $72(83)$ \\
Disco & $16(18)$ & - & $74(85)$ \\
Gentamicina & \\
E test & $15(17)$ & & \\
Disco & $13(15)$ & - & \\
Estreptomicina*** & & & \\
E test & &
\end{tabular}

* Breakpoints utilizados para sensibilidade e resistência, respectivamente: teicoplanina: $\leq 8 \mu \mathrm{g} / \mathrm{mL}$ e $\geq 32 \mu \mathrm{g} / \mathrm{mL}$ ou $\geq 14 \mathrm{~mm}$ e $\leq 10 \mathrm{~mm}$; ampicilina: $\leq 8 \mu \mathrm{g} / \mathrm{mL}$ e $\geq 16 \mu \mathrm{g} / \mathrm{mL}$ ou $\geq 14 \mathrm{~mm}$ e $\leq 10 \mathrm{~mm}$; penicilina: $\leq 8 \mu \mathrm{g} / \mathrm{mL}$ e $\geq 16 \mathrm{mg} / \mathrm{ml}$ ou $\geq 15 \mathrm{~mm}$ e $\leq 14 \mathrm{~mm}$; gentamicina: $\leq 500 \mu \mathrm{g} / \mathrm{mL}$ e $>500 \mu \mathrm{g} / \mathrm{mL}$ ou $\geq 10 \mathrm{~mm}$ e $\leq 6 \mathrm{~mm}$; estreptomicina: $\leq 2.000 \mu \mathrm{g} / \mathrm{mL}$ e $>2.000 \mu \mathrm{g} / \mathrm{mL}$ ou $\geq 10 \mathrm{~mm}$ e $\leq 6 \mathrm{~mm}$.

** MIC, microdilução em placas.

** Sessenta e uma amostras (70\%) mostram alto grau de resistência (high-level), tanto para gentamicina quanto para estreptomicina.

Como o enterococo apresenta intrinsecamente baixo grau de resistência a aminoglicosídeos e os antimicrobianos dessa classe não são ativos contra amostras desse gênero quando utilizados isoladamente, torna-se importante a avaliação do alto grau de resistência a aminogl i cosídeos. Esse mecanismo de resistência é adquirido pela bactéria (não intrínseco) e leva a perda do sinergismo com antimicrobianos que agem na parede bacteriana ( $\beta$ lactâmi cos e glicopeptídeos). Sem esse sinergismo, não ocorrerá ação bactericida, dificultando muito a erradicação de infecções graves. O NCCLS define como alto grau de resistência MICS superiores a $500 \mu \mathrm{g} / \mathrm{mL}$ para gentamicina e MICS superiores a $2.000 \mu \mathrm{g} / \mathrm{mL}$ para estreptomicina. Só é necessária a avaliação destes dois aminoglicosídeos, uma vez que amostras que apresentam al to grau de resistência à gentamicina irão apresentar também alto grau de resistência a todos os aminoglicosídeos, podendo ser exceção apenas a estreptomicina ${ }^{7}$.

\section{RESULTADOS}

A tabela 1 mostra os resultados obtidos na avaliação dos antimicrobianos normalmente utilizados no tratamento de infecções enterocócicas. Baseando-se nos resultados do $\mathrm{E}$ test, $86 \%$ dos isolados foram resistentes à ampi cilina eà penicilina no presente estudo. A mai or parte das amostras resis- 


\begin{tabular}{|c|c|c|c|}
\hline \multirow{2}{*}{ Antimicrobianos } & \multicolumn{3}{|c|}{ Número $(\%)^{\star \star}$ de isolados sensíveis } \\
\hline & $\begin{array}{l}\text { E. faecalis } \\
\qquad(n=76)\end{array}$ & $\begin{array}{l}\text { E. faecium } \\
(n=11)\end{array}$ & $\begin{array}{l}\text { Total } \\
(n=87)\end{array}$ \\
\hline Teicoplanina & $10^{* * *}(91)$ & $14(18)$ & $25(29)$ \\
\hline Ampicilina & $11(100)$ & $1(1)$ & $12(14)$ \\
\hline Penicilina & $9(82)$ & $2(3)$ & $11(13)$ \\
\hline Gentamicina & $0(0)$ & $16(21)$ & $16(18)$ \\
\hline Estreptomicina & $0(0)$ & $13(17)$ & $13(15)$ \\
\hline \multicolumn{4}{|l|}{ Gentamicina ou } \\
\hline estreptomicina & $0(0)$ & $26(34)$ & $26(30)$ \\
\hline Nenhum ${ }^{\star * * *}$ & $0(0)$ & $42(55)$ & $42(48)$ \\
\hline \multicolumn{4}{|c|}{$\begin{array}{l}\text { * Baseados nos resultados do E-test. } \\
\text { ** Baseados no número total de amostras de cada espécie. } \\
\text { *** Sete dessas amostras apresentam padrão cromossômico idêntico } \\
\text { quando avaliados pela técnica de pulsed-field gel electrophoresis. } \\
\text { ***Amostras resistentes aos } 5 \text { antimicrobianos (vancomicina, teicopla- } \\
\text { nina, ampicilina, penicilina, gentamicina e estreptomicina). }\end{array}$} \\
\hline
\end{tabular}

tentes aos $\beta$-lactâmicos era de $\mathrm{E}$. faecium (sensibilidade de $1 \%$ a ampicilina - tabela 2). Para E. faecalis obtivemos $100 \%$ de sensibilidade a ampicilina e $82 \%$ a penicilina (tabela 2). Essa observação corrobora os dados da literatura, que mostram a preponderância de cepas de E. faecium resistentes aos $\beta$-lactâmicos quando comparados aos isolados de $\mathrm{E}$. faecal is $\mathrm{s}^{4}$. $\mathrm{Na}$ verdade, o encontro de amostras de $\mathrm{E}$. faecalis resistentes à ampicilina é bastante raro.

Em relação aos aminoglicosídeos, a percentagem de al to grau de resistência foi de $82 \%$ e $85 \%$ para gentamicina e estreptomicina, respectivamente, com $69 \%$ dos isolados (61 amostras) apresentando alto grau de resistência aos dois aminoglicosídeos testados e, conseqüentemente, a todos os aminoglicosídeos. Finalmente, para teicoplanina, observamos taxa de sensibilidade de $25 \%$, vaIor semelhante ao encontrado por Willey et al. ( $70 \%$ de resistência a teicoplanina entre seus isolados de ERV) ao avaliar 155 amostras de enterococos enviados de vários centros² .

Quanto aos antimi crobianos alternativos analisados, os que apresentaram maiores taxas de resistência foram eritromicina, trimetoprim-sulfametoxazol e ciprofloxacina $(98 \%, 83 \%$ e $69 \%$, respectivamente). Por outro lado, observamos maior sensibilidade a cloranfenicol, doxiciclina e trospectomicina $(82 \%, 92 \%$ e $94 \%$ de isolados sensíveis, respectivamente) [tabela 3].

Houve excelente concordância entre os testes de sensibilidade analisados. As amostras foram testadas pel as três metodologias (microdiluição em placa, E test e disco difusão) somente para teicoplanina e houve apenas três resultados discordantes entre mi crodiluição em placa e $E$ test $(96,6 \%$ de concordân-
Tabela 3 - Padrão de sensibilidade ${ }^{\star}$ de 87 amostras de ERV a drogas alternativas

\begin{tabular}{|c|c|c|c|}
\hline Antimicrobianos & $\begin{array}{l}\text { Sensíveis } \\
\text { n (\%) }\end{array}$ & $\begin{array}{l}\text { Intermediárias } \\
\text { n (\%) }\end{array}$ & $\begin{array}{c}\text { Resistentes } \\
\text { n (\%) }\end{array}$ \\
\hline \multicolumn{4}{|l|}{ E test } \\
\hline Cloranfenicol & $71(82)$ & $13(15)$ & $3(3)$ \\
\hline Doxiciclina & $80(92)$ & $3(3)$ & $4(5)$ \\
\hline Esparfloxacina** & $48(55)$ & -- & $39(45)$ \\
\hline \multicolumn{4}{|l|}{ Disco-difusão } \\
\hline Ciprofloxacina & $3(3)$ & $24(28)$ & $60(69)$ \\
\hline Clinafloxacina** & $30(34)$ & $19(22)$ & $38(44)$ \\
\hline Eritromicina & -- & $2(2)$ & $85(98)$ \\
\hline Espectinomicina** & $12(14)$ & $56(64)$ & $19(22)$ \\
\hline Trospectomicina*** & $82(94)$ & - & $5(6)$ \\
\hline $\begin{array}{l}\text { Sulfametoxazol- } \\
\text { trimetoprim }\end{array}$ & $5(6)$ & $10(11)$ & $72(83)$ \\
\hline \multicolumn{4}{|c|}{$\begin{array}{l}\text { * Os halos de inibição para novobiocina foram de } 16 \text { a } 31 \mathrm{~mm} \text { (média de } 27 \text { ). } \\
\text { ** Breakpoints de sensibilidade e resistência (respectivamente): es- } \\
\text { parfloxacina, } \leq 2 \mu \mathrm{g} / \mathrm{mL} \text { e } \geq 8 \mu \mathrm{g} / \mathrm{mL} \text {; clinafloxacina, } \geq 23 \mathrm{~mm} \text { e } \leq 19 \mathrm{~mm} \text {; } \\
\text { espectinomicina, } \geq 18 \mathrm{~mm} \text { e } \leq 14 \mathrm{~mm} \text {. }\end{array}$} \\
\hline
\end{tabular}

cia). Três amostras foram classificadas como intermediária pelo $\mathrm{E}$ test e sensível pela técnica de microdiluição. As amostras foram testadas pelas técnicas de $\mathrm{E}$ test e disco difusão contra ampicilina, teicoplanina, vancomicina egentami cina, ea taxa de concordância foi de 98\% (tabela 1).

\section{DISCUSSÃO}

Enterococos resistentes a vancomicina foram originalmente descritos na Europa Ocidental, em 1988. A partir dessa época, começaram a surgir relatos de seu aparecimento em vários países do mundo, incluindo Estados Unidos ${ }^{11,12}$. A resistência à vancomicina tem sido observada principalmente entre quatro espécies de enterococos: $E$. faecalis, E. faecium, E. avi um e E. gallinarum ${ }^{13}$. A base genética para essa resistência já está bem estudada e envolve vários determinantes gênicos encontrados em transposomos, dois componentes do sistema regulatório (vanR e vanS), uma desidrogenase (vanH) e uma ligase que condensa Dalanina com o produto do vanH para produzir precursores peptidoglicanos (vanA). Fenotipicamente, esses enterococos caracterizam-se por alto nível de resistência à vancomicina (MIC $\geq 120 \mu \mathrm{g} /$ $\mathrm{mL}$ ) e tei coplanina (MIC $\geq 16 \mu \mathrm{g} / \mathrm{mL}$ ). Esse fenótipo é chamado VanA. Outro fenótipo de resistência a glicopeptídeos caracteriza-se por resistência à vancomicina, mas sensibilidade à teicoplanina e pela presença do gene vanB ( $E$. faecium e $E$. faecalis) ou do gene vanC (E. gallinarum) ${ }^{4}$. No presente estudo, 29\% das amostras resistentes à vancomicina foram sensíveis à teicoplanina (fenó- 
tipo VanB), mostrando que esse antimicrobiano pode ser muitoútil em locais onde há altas taxas de resistência à vancomicina.

Quanto aos antibióticos $\beta$-lactâmicos, o mecanismo de resistência dos enterococos se deve a alterações nas proteínas ligadoras de penicilina (PBPS). As PBPS dos enterococos resistentes apresentam afinidade diminuída à penicilina, que chega a ser 10 a 100 vezes menor que àquel a apresentada pel as PBPS dos estreptococos. I sso se torna mais evidente para as penicilinas semi-sintéticas resistentes à penicilinase, tais como nafcilina ( $\mathrm{MIC}=8-50 \mu \mathrm{g} / \mathrm{mL})$ e meticilina $(\mathrm{MIC} \geq 50 \mu \mathrm{g} / \mathrm{mL})^{14}$. Como pudemos ver neste estudo, a grande maioria das amostras $\mathrm{E}$. faecium resistentes à vancomicina tende a ser resistente também aos $\beta$-lactâmi cos (tabel a 2 ). Por outro lado, amostras de $\mathrm{E}$. faecalis tendem a ser sensíveis aos $\beta$-lactâmicos, independentemente do padrão de sensibilidade a glicopeptídeos.

Ultimamente, têm sido descritos enterococos produtores de $\beta$-lactamases ${ }^{15}$. No entanto, essa resistência pode não ser detectada por testes de sensibilidade de rotina devido ao efeito de inóculo, isto é, pequenas variações de inóculo podem levar a grandes variações da MIC. Felizmente, esse mecanismo de resistência é raro, sendo encontrado em apenas $1 \%$ a $2 \%$ das cepas resistentes à ampicilina ${ }^{3}$. A produção de $\beta$-lactamase não foi detectada em nenhuma das amostras avaliadas neste estudo.

Os enterococos apresentam baixo grau de resistência aos aminoglicosídeos. Essa é uma característica intrínseca do gênero, porém esses antimicrobianos apresentam efeito sinérgico quando associados a $\beta$-lactâmicos ou glicopeptídeos. Têm surgido, no entanto, enterococos que expressam alto grau de resistência aos aminoglicosídeos. O alto grau de resistência (high level) a aminoglicosídeos é decorrente da produção de enzimas modificadoras de aminoglicosídeos, tais como 6'-acetiltransferase (AAC-6') e 2"-fosfotransferase (APH-2"). Com exceção da AAC-6' do E. faecium que é cromossomicamente codificada, os genes correspondentes às demais enzimas são localizados em plasmídeos ${ }^{15}$. A presença de alto grau de resistência a aminoglicosídeos leva a perda de sinergismo com $\beta$-lactâmicos e glicopeptídeos, não se conseguindo, dessa maneira, efeito bactericida ${ }^{14}$. Este estudo mostrou que as taxas de alto grau de resistência a aminoglicosídeos parecem ser bastante al tas (tabela 1), o que dificulta muito o tratamento de infecções graves causadas por essas cepas resistentes, uma vez que a presença de alto grau de resistência a aminoglicosídeos significa que não será conseguido efeito bactericida.
Como pode ser avaliado no presente estudo, infecções graves causadas por enterococos resistentes à vancomicina serão normalmente de controle muito difícil, principalmente quando se tratar de $\mathrm{E}$. faecium, uma vez que amostras dessa espécie apresentaram altas taxas de resistência a $\beta$-lactâmicos e aminoglicosídeos. Na maioria das vezes, a única opção terapêutica com eficácia comprovada em estudos clínicos parece ser a teicoplanina ${ }^{16}$. Além disso, estudos realizados com amostras clínicas isoladas em hospitais brasileiros mostram excelente atividade in vitro desse antimicrobiano ${ }^{17}$.

Alguns dos antibióticos alternativos analisados apresentaram boa atividade in vitro contra enterococos, porém não sabemos o verdadeiro impacto desses resultados na prática médica. U m exemplo é o que foi observado em rel ação ao novo aminociclitol testado, a trospectomicina. Essa droga, o mais potente derivado da espectinomicina, é inativada pelas mesmas enzimas que destroem a estreptomicina e a gentamicina e, portanto, o resultado obtido in vitro ( $94 \%$ de sensibilidade) deve representar falsa sensibilidade ${ }^{10}$.

Cloranfenicol e tetraciclina são antimicrobianos para os quais é comum observar-se sensibilidade dos enterococos, mesmo para cepas resistentes a todos os outros antimicrobianos. No entanto, no passado já se tentou utilizar o cl oranfenicol no tratamento desses patógenos, sem êxito ${ }^{4}$. As tetraciclinas também não parecem apresentar resultados clínicos muito bons, especialmente em infecções potencialmente graves 4 .

Agentes de várias classes, incluindo fluoroquinolonas, estreptograminas e novobiocina, em baixas concentrações, têm sido testados contra enterococos e têm demonstrado inibir o crescimento in vitro desse microrganismo ${ }^{4}$. A novobiocina, um derivado cumarínico diidroxiglicosilado, já foi utilizada previamente para o tratamento de infecções por microrganismos gram-positivos ${ }^{18}$. Devido à sua rel ativa toxici dade è al ta taxa de surgimento de resistência, ela foi gradativamente abandonada. Contudo, quando começaram a surgir estafilococos resistentes à meticilina, a utilização desse antibiótico voltou a ser avaliada. Para enterococos, no entanto, dados disponíveis até o momento não são suficientes para analisar sua eficácia e há trabalhos demonstrando apenas atividade modesta contra esses patógenos. Além disso, a ligação protéica dessa droga é muito alta, o que pode interferir na sua eficácia in vivo ${ }^{18,19}$.

Uma outra classe de agentes antimicrobianos que demonstra atividade in vitro contra enterococos é o grupo das fluoroquinolonas. No entanto, tem-se observado emergência de resi stência, tanto 
in vivo quanto in vitro ${ }^{20}$. À semelhança da novobiocina, esses compostos bloqueiam a ação da DNA-girase, e a associação dessas duas drogas pode vir a representar uma boa opção no tratamento de infecções por enterococos ${ }^{20,21}$. As novas quinolonas têm demonstrado boa atividade contra enterococos, em especial a clinafloxacina, cujo radical pirrolidinil na posição 7 garante maior atuação contra bactérias gram-positivas ${ }^{9}$, e a esparfloxacina, um membro difluorinatado do ácido quinolonacarboxílico. Em trabalho publicado por J ones et al., a concentração de antimicrobiano necessária para inibir $50 \%$ das amostras de enterococos estudadas (MIC 50) foi de $0,5 \mu \mathrm{g} / \mathrm{mL}$ e a concentração necessária para inibir $90 \%$ das amostras (MIC 90) foi $2 \mu \mathrm{g} / \mathrm{mL}$. As MICS variaram de 0,12 a $2 \mu \mathrm{g} / \mathrm{mL}^{22}$.

A análise comparativa dos testes de sensibilidade demonstrou que o teste de disco difusão pode apresentar bons resultados quando utilizado adequadamente. Porém, esse teste deve ser realizado com muita cautela e controle rígido de qualidade; do contrário, poderá falhar na detecção de amostras de enterococos resistentes ${ }^{23}$. Os resultados do presente estudo também mostraram, em concordância com outros trabal hos já publicados, que o $\mathrm{E}$ test é uma excelente metodologia para a avaliação da sensibilidade de enterococos a antimi crobianos. Além de apresentar alta acurácia, é um teste de fácil realização e que fornece, com precisão, o $\mathrm{MIC}^{3,24}$. Além disso, as fitas para os cinco antimicrobianos que necessitam ser testados para enterococos podem ser col ocadas em uma única placa grande de Muller-Hinton.

$\mathrm{O}$ tratamento dos enterococos multirresistentes ainda é um desafi o e vários esquemas já vêm sendo propostos na literatura, a maioria dos quais utilizando fluoroquinolonas mas com resultados bastante contraditórios ${ }^{21}$. São necessários, então, mais trabalhos analisando a efetividade dessas combinações de anti bi óticos antes que recomendações definitivas possam ser feitas.

\section{SUMMARY}

\section{Antimicrobial susceptibility testing of 87 clinical isolates of vancomycin-resistant enterococci}

Oвj ectives. 1) To evaluate the antimicrobial susceptibility pattern of vancomycin-resistant enterococci to the antimicrobial agents that are commonly used to treat enterococci infections and to some al ternative drugs. 2) To eval uate the accuracy of $E$ test for susceptibility testing enter ococci.

Material and Method. We evaluated 87 clinical
VRE isolates that were selected from a previous study which analyzed 1936 clinical isolates collected and processed in 97 us medical centers in the last quarter of 1992. The isolates were identified to the species level by using the API 20S System, the Vitek gram-positive identification cards and a modified version of the conventional method proposed by Facklam and Collins. The in vitro susceptibility testing was performed by broth microdilution, E test and disk diffusion methods, following the criteria described by the National Committee for Clinical Laboratory Standards (NCCLS). TheVRE isolates were tested against antimicrobial agents commonly used to treat enterococci infections (vancomycin, teicoplanin, ampicillin, penicillin, gentamicin and streptomycin) and against ten potential alternative drugs (chloramphenicol, doxycycline, sparfloxacin, ciprofloxacin, clinafloxacin, erythromycin, spectinomycin, trospectomycin, trimetoprim-sulfametoxazol and novobiocin).

RESULTS. Our results showed a high rate of resistance to ampicillin and penicillin (86\%). High level resistance to gentamicin and streptomycin was demonstrated by $82 \%$ and $85 \%$ respectively. Although teicoplanin and vancomycin belong to the same antibiotic group (glycopeptide), $29 \%$ of VRE were susceptible to teicoplanin.

Among the alternative drugs, trospectomycin, doxycyclin and chloramphenicol showed the highest in vitro activity, with $94 \%, 92 \%$ and $82 \%$ susceptibility respectively. In addition, erythromycin, trimetoprim-sulfametoxazol and ciprofloxacin showed the highest rates of resistance $(98 \%$, $83 \%$ and $69 \%$, respectively).

Conclusion. The treatment options for infections caused by vancomycin-resistant enterococci seem to be very narrow since a small percentage of those i sol ates were suscepti ble to the other antimicrobial agents commonly used to treat these infections and only a few of the al ternative drugs tested showed good in vitro activity. Many regimens using various antibiotic combinations have been tested against VRE, most of them with fluoroquinol ones. However further studies are necessary to evaluate the clinical role of these antibiotic combinations. [Rev Ass Med Brasil 1997; 43(3); 217-22.]

KEY WORDS: Vancomycin-resistant enterococci. In vitro antimicrobial activity. Antimicrobial resistance. New antimicrobial agents. E test.

\section{REFERÊNCIAS BIBLIOGRÁFICAS}

1. Noskin GA, Peterson LR, Warren J R. Enterococcus faecium and Enterococcus faecal is bacteremia: acquisition and outocome. Clin Infect Dis 1995; 20: 296-301.

2. Willey BM, McGeer AJ, Ostrowski MA, Kreiswirth BN, Low 
DE. The use of molecular typing techniques in the epidemiologic investigation of resistant enterococci. Infect Control Hosp E pidemiol 1994; 15: 549-55.

3. J ones RN, Sader HS, Erwin M, Anderson SC and the Enterococcus Study Group. E merging multiply resistant enterococci among clinical isolates: prevalence data from 97 medical center. Surveillance study in the United States. Diag Microbiol Infect Dis 1995; 21: 85-93.

4. Eliopoul os GM . Increasing problems in the therapy of enterococcal infections. Eur J Clin Microbiol Infect Dis 1993; 12: 40912.

5. Buschelman BJ, Bale MJ, J ones RN. Species identification and determination of high-level resistance among enterococci. Comparison study of sterile body fluid isolates, 19851991. Diagn Microbiol Infect Dis. 1993; 16: 119-22.

6. Facklam RR, Collins MD. I dentification of enterococcus species isolates from human infections by a conventional test scheme. J Clin Microbiol 1989; 27: 731-4.

7. National Committee for Clinical Laboratory Standards. Methods for dilution antimicrobial susceptibility test for bacteria that grow aerobically. Approved standard, M7-A3. 1993. National Committeefor Clinical Laboratory Standards, Villanova, PA.

8. National Committee for Clinical Laboratory Standards. Performance standards for antimicrobial disk susceptibility tests. Approved standard, M2-A5. 1993. National Committee for Clinical Laboratory Standards, Villanova, PA.

9. Burney S, Landman D, Quale J M. Activity of clinafloxacin against multidrug-resistant Enterococcus faecium. Antimicrob Agents Chemother 1994; 38: 1.668-70.

10. Barry AL, J ones RN, Thornsberry C. Antibacterial activity of trospectomycin (U- 63366F) and initial evaluations of disk diffusion susceptibility tests. Antimicrob Agents Chemother 989; 33: 569-72.

11. Gutmann L, Billot-Klein D, Al-ObeidS et al. Induciblecarboxypeptidase activity in vancomycin-resistant enterococci. Antimicrob Agents Chemother 1992; 36: 77-80.

12. Sader HS, Pfaller MA, Tenover FC, Hollis RJ, J ones RN. Evaluation and characterization of multiresistant Entero- coccus faecium from twelve U.S. medical centers. J Clin Microbiol 1994; 32: 2.840-2.

13. J ohnson AP, Uttley AHC, Woodford N, George RC. Resistance to vancomycin and teicoplanin: a emerging clinical problem. Antimicrob Agents Chemother 1990; 34: 280-91.

14. Patterson J E, Wanger A, Zscheck KK, Zervos MJ , Murray BE. Molecular epidemiology of $\beta$-lactamase producing enterococci. Antimicrob. Agents Chemother 1990; 34: 302-5.

15. Leclercq R, Dutka-Malen S, Brisson-N oel A et al. Resistance of enterococci to aminoglycosides and glycopeptides. Clin Infect Dis 1992; 15: 495-501.

16. Phillip G. Vancomycin and teicoplanin: something old, something new. Med J Aust 1992; 156: 53-7.

17. Mimica I, Mendes CM, Mimica L et al. Estudo da sensibilidade de Staphylococcus sp. e Enterococcus sp. à teicoplanina e à vancomicina. Rev Ass Med Brasil 1996; 42: 147-50.

18. French $P$, Venuti $E$, Fraimow HS. In vitro activity of novobiocin against multiresistant strains of Enterococcus faecium. Antimi crob Agents Chemother 1993 37: 2.736-9.

19. J ones RN. Should novobiocin be clinically re-evaluated? Diagn Microbiol Infect Dis 1989; 12: 363-5.

20. Nakanishi N, Yoshida S, Wakebe H, Inoue M, Mitsuhashi S. Mechanisms of clinical resistance to fluoroquinol ones in $\mathrm{En}$ terococcus faecalis. Antimicrob Agents Chemother 1991; 35: 1.053-9.

21. Landman D, Mobarakai NK, QualeJ M. Novel antibiotic regimens against Enterococcus faecium resistant to ampicillin, vancomycin and gentamicin. Antimicrob Agents Chemother 1993; 37: 1.904-8.

22. J ones RN, Barrett MS, Erwin ME, Briggs BM, J ohnson DM. In vitro antimicrobial activity of sparfloxacin (AT-4140, CI-978, PD 131501) compared with numerous other quinolone compounds. Diagn Microbiol Infect Dis 1991; 14: 319-30.

23. Tenover FC, Tokars J, Swenson J et al. Ability of clinical laboratories to detect antimicrobial agent-resistant enterococci. J Clin Microbiol 1993; 31: 1.695-9.

24. Sader HS, Pignatari AC. E test: a novel techniquefor antimicrobial susceptibility testing. Sao Paulo Med J 1994; 12: 635-8. 